Results There was a significant reduction in admission rates after institution of the new policy. The proportion of hospital admissions to PED observation unit cases was significantly reduced as a whole from $64.9 \% \pm 5.1 \%$ to $33.2 \pm 0.6 \%$ and also for the common paediatric problems studied.

Conclusion A multidisciplinary paediatric emergency department policy, using as much available evidence as possible, was successful in significantly reducing paediatric hospital admissions.

\section{PS-346 IMPROVING THE STANDARD OF INVASIVE PROCEDURES IN PAEDIATRICS - A TEACHING INTERVENTION TO ALIGN CURRENT PRACTICE TO BRITISH PSYCHOLOGICAL SOCIETY GUIDELINES}

M Monaghan, J Criddle, A Osborn. Paediatric Emergency Department, Evelina London Children's Hospital, London, UK

\subsection{6/archdischild-2014-307384.645}

Background/aims Invasive procedures (eg intravenous cannulation) remain a leading cause of distress in children. They are performed daily yet rarely taught formally, leading to variation in practice. The British Psychological Society (BPS) created guidelines in 2010, although these have yet to be implemented in a formal teaching programme. Our teaching intervention aimed to align practice to BPS guidelines and improve professional confidence.

Methods Teaching sessions were delivered to professionals involved in cannulation at two South London hospitals by a senior play specialist and a paediatrician. Sessions covered assessment, preparation, teamwork, implementation and post-procedural care. A poster containing a flowchart was used for teaching and departmental reference.

Results Thirteen professionals involved in cannulation $(9$ medical, 4 nursing) reported their previous teaching experiences. 5 reported never receiving training in paediatric invasive procedures, 6 informal teaching and only 2 receiving formal teaching (1 medical, 1 nursing). Pre-intervention confidence was 5.7/10 $(n=13)$ [range 0-8]. This improved to 7.3/10 [range 4-9] post intervention. The intervention usefulness was rated $8.3 / 10(\mathrm{n}=$ 12 , range 5-10]. When self-reporting practical considerations for cannulation, 8/12 mentioned local anaesthetic creams but only 6/ 12 mentioned using distraction or a play specialist.

Conclusions This intervention aimed to address the lack of formal teaching available in Paediatric invasive procedures. It highlighted areas of BPS guidance that remain underappreciated despite their greater efficacy (eg the importance of distraction vs anaesthetic cream). Initial levels of confidence showed improvement post-intervention. We recommend this teaching intervention to be used regularly throughout Paediatric departments and undergraduate medical programmes.

\section{PS-347 HEALTH-RELATED QUALITY OF LIFE OF PORTUGUESE CHILDREN AND ADOLESCENTS IN PRIMARY CARE: REGIONAL CHARACTERISTICS AND COMPARISON TO EUROPEAN DATA}

${ }^{1}$ I Marques, ${ }^{2} \mathrm{M}$ Abreu, ${ }^{1} \mathrm{C}$ Prelhaz, ${ }^{2} \mathrm{M}$ Martins, ${ }^{1} \mathrm{AG}$ Silva, ${ }^{3} \mathrm{TM}$ Fernandes, ${ }^{4} \mathrm{R}$ Ascenção, ${ }^{3} \mathrm{P}$ Gomes. ${ }^{1}$ Paediatrics, Centro Hospitalar Barreiro-Montijo, Barreiro, Portugal; ${ }^{2}$ Paediatrics, Hospital Pedro Hispano, Matosinhos, Portugal; ${ }^{3}$ Family Medicine, Unidade Saúde Familiar Infesta, Matosinhos, Portugal; ${ }^{4}$ Family Medicine, Unidade Cuidados Saúde Personalizados Sete Rios, Lisboa, Portugal
Background Children and adolescent health has been recognised as an important indicator of a country's development and global health. Adopting a health-related quality of life (HRQoL) approach can not only increase understanding of their own health but also help to establish policies that promote their safety and wellbeing.

Aims Evaluate HRQoL in a sample of Portuguese children and adolescents at primary care level, and assess age, gender and regional differences. Compare these results with the available European data.

Methods Cross-sectional observational study. Participants included 163 children and adolescents aged 8-18 years, presenting to a regular follow-up consultation at two primary care services (Lisbon and Oporto), during a six months period between 2012/13. HRQoL was assessed using KIDSCREEN-27, a selfreport questionnaire that consists of five dimensions: Physical Wellbeing, Psychological Wellbeing, Autonomy and Parent Relations, Social Support and Peers, and School Environment. Statistics were calculated using SPSS Statistics 20.0.

Results The HRQoL scores, in all five dimensions, were consistently lower in female gender, adolescents and patients from Oporto region. However, statistically significant differences were found only for gender and age at the physical wellbeing dimension ( $p=0.001$ and $p=0.02$, respectively). Compared to the European data our patients presented significantly higher HRQoL scores in all five dimensions ( $\mathrm{p}=0.025)$.

Conclusions The results underline the fact that females and adolescents should be the main targets when planning successful health policies and practices. Also, they call the attention for loco-regional differences that must be taken into account. Despite our patients presenting higher HRQoL scores, the results are according to the literature.

\section{PS-348 CHILDREN'S CLINICAL PROFILE: TERTIARY PAEDIATRIC BALANCE CLINIC}

${ }^{1}$ A Shetye, ${ }^{2}$ E Raglan. 'Department of Audiological Medicine, East and North Herts NHS Trust, Stevenage, UK; ${ }^{2}$ Department of Audiological Medicine, Great Ormond Street Hospital NHS Foundation Trust, London, UK

\subsection{6/archdischild-2014-307384.647}

Main purpose The main aim was to analyse the clinical profile of children with and without hearing impairment referred with various vestibular related symptoms attending a tertiary balance clinic over six months. Children undergoing pre-cochlear implant assessment were also included.

Design of study A thorough clinical history and evaluation of auditory and vestibular assessment was undertaken. Diagnosis of the medical condition was made on the basis of all the above.

Results One hundred and twenty four consecutive patients (55 female and 69 male) were included. The age range was 9 months to 18.5 years (mean age 9.41 years). Multiple professionals referred these patients.

Associated conditions: Ten patients had a history of migraine, 8 had cyclical vomiting, 8 had CHARGE syndrome, 7 referred post-meningitis had labyrinthitis ossificans. Three patients sustained head injury.

Signs:- Two patients had positional nystagmus: one with history of head injury, the other sustained vibration injury to the labyrinth caused during fairground rides. Head impulse test was positive bilaterally in seventeen patients, indicated by the presence of catch-up saccade, a clinical feature of vestibular failure. 
Objective Vestibular assessment:- Electronystagmography showed central vestibular abnormalities in 18 (14.5\%), 66 had normal vestibular function and 41 had vestibular hypofunction. Seventeen had bilateral vestibular failure.

Implications and relevance of results In children, numerous aetiologies of dizziness and balance problems may have a similar clinical picture. The symptoms of non-vestibular disorders can masquerade those of vestibular disorders and vice-versa. Close multispecialty working is vital for positive clinical outcome.

\section{PS-349 TELL ME HOW YOU SLEEP: SLEEP HABITS AND DISTURBANCES IN PORTUGUESE PRE-SCHOLAR AND SCHOLAR CHILDREN}

S Lopes, F Almeida, S Jacob, M Figueiredo, C Vieira, F Carvalho. Pediatrics, Centro Hospitalar Do Médio Ave E. P. E., Vila Nova de Famalicão, Portugal

\subsection{6/archdischild-2014-307384.648}

Background and aims Given the risk of significant medical and behavioural morbidity, it is crucial to evaluate sleep disorders in clinical practice. We aimed to evaluate sleep habits and disturbances in pre-scholar and scholar children from a Northern region of Portugal and compare the results with other studies.

Methods Children's Sleep Habits Questionnaire Portuguese version (CSHQ-PT) was applied to parents of healthy children (410 years old) attending daycare centres and elementary schools in our city.

Results 107 pre-scholar and 122 scholar children were included $(n=229)$, mean age 6,3 years. 54,1\% were boys. Prevalence of co-sleeping was 37,3\%. Mean bedtime was 9:41 pm and mean wake-up time was 7:20 am. Mean sleep duration was $9.7 \mathrm{~h}$, being longer in pre-scholars $(p<0.001) .75,7 \%$ of children suffered from global sleep disturbances and the mean CSHQ-PT total score was 47,05 , being higher in pre-scholars $(\mathrm{p}=0,001)$. Pre-scholars also scored higher on bedtime resistance $(\mathrm{p}<$ $0,001)$, sleep onset delay $(\mathrm{p}=0,046)$ and night wakening $(\mathrm{p}<$ $0,001)$. Bedtime resistance and sleep anxiety scores were higher in only children $(\mathrm{p}=0.003)$. The prevalence of enuresis was $7,7 \%$, restless sleep 52,7\% and bruxism 22,2\%. Pre-scholars scored higher in night terrors $(p=0,04)$. It was hard to get the children out of bed in $56.7 \%$ and the children seemed tired during the day in $22.9 \%$.

Conclusion Included children tend to fall asleep later and sleep less than in other studies. A high prevalence of sleep disturbances was found, specially in pre-scholar children, which claims a different approach of this subject in our clinical practice.

\section{PS-350 WITHDRAWN}

\section{PS-351 A GROWING PAINS (GP) QUESTIONNAIRE : VALIDATION AND RELIABILITY CONTROL}

${ }^{1} \mathrm{M}$ Vasilopoulou, ${ }^{2} \mathrm{E}$ Kritseli, ${ }^{2} \mathrm{~N}$ Myriokefalitakis, ${ }^{2} \mathrm{M}$ Tsolia. ${ }^{2}$ 2nd Pediatric Clinic/PICU Penteli'sChildren Hospital, University of Athens Medical School, Athens, Greece; ${ }^{2} 2 n d$ Pediatric Clinic and a Kyriakou Children's Hospital, University of Athens Medical School, Athens, Greece

\subsection{6/archdischild-2014-307384.649}

Background Growing pains (GP) is a common cause for visiting the paediatrician. Their prevalence remains obscure and a screening questionnaire is not available.
Aim To develop a questionnaire, appropriate for the estimation of GP prevalence in the general children's population aged 4-7 years old.

Material and methods After review of the literature, a 27-closed type questions with two-answer options (yes-no) questionnaire was developed, including the parameters of localization, duration, intensity and heredity of growing pains. Content validity and test-retest have been examined in a pilot study preceding the present study. The validity of the questionnaire was further studied in a parental population of children aged 4-7 years old, sourced from a randomly selected elementary school in Athens. The reliability was examined by repeated parents response over 3 weeks.

Results 83 questionnaires were filled out. The final version of the questionnaire comprised 21 questions. The mean test-retest reliability was $75.4 \%$, ranging from $0.33-100 \%$ ( $\mathrm{p}<0.001$, Cronbach alpha coefficient was equal to 0.68 for total scale, ranging from $0.62-0.82$ for the subscales). Construct validity evaluation defined 9 fields incorporating the 21 questions. After excluding low reliability questions, nine items remained in the scale. A cut-off point of $\geq 8$ was finally proposed for the detection and diagnosis of growing pains.

Conclusions We report the development of a reliable and valid questionnaire for GP based on the existing publications. This questionnaire focused on the domains of pain localization, intensity of growing pains and their $24 \mathrm{~h}$ temporal distribution.

\section{PS-352 CHARACTERISTICS OF U. S. CHILDREN WHOSE PARENTS REPORT THEY NO LONGER HAVE AUTISM SPECTRUM DISORDER (ASD)}

${ }^{1} \mathrm{G}$ Montes, ${ }^{2} \mathrm{JS}$ Halterman. ${ }^{1}$ Ralph C. Wilson Jr. School of Education, St. John Fisher College/Children's Institute Inc., Rochester, USA; ${ }^{2}$ Department of Pediatrics, University of Rochester, Rochester, USA

\subsection{6/archdischild-2014-307384.650}

Background/aims Our objective was to determine the characteristics of children who do not retain an autism diagnosis.

Methods The National Survey of Children's Health 2007, a nationally representative survey, asked parents whether a doctor or other health professional had ever told them their child had ASD. Thirty eight percent of those who responded affirmatively also reported that the child did not have ASD currently. We inspect the differences between the ASD-now and no-longer-

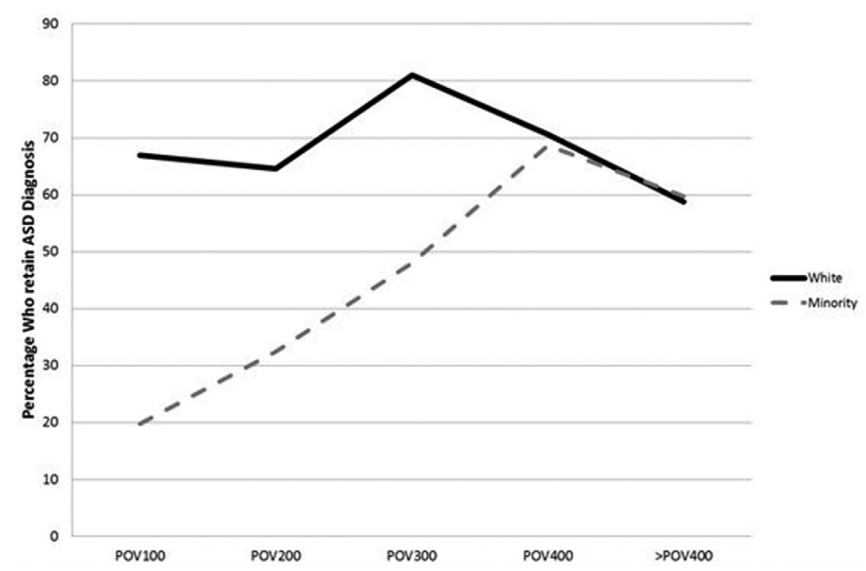

Abstract PS-352 Figure 1 Percentage of children who retain ASD diagnosis by income level and race 